\title{
Anthocyanin-Rich Purple Corn Extract and Its Effects on the Blood Pressure of Adults
}

Journal of Evidence-Based

Complementary \& Alternative Medicine 18(4) 237-242

(C) The Author(s) 2013

Reprints and permission: sagepub.com/journalsPermissions.nav DOI: 10.1 I77/2। 565872।3482942 cam.sagepub.com

(S)AGE

\author{
Madelon L. Finkel, PhD', Sixto Sanchez, MD, MPH ${ }^{2}$, \\ Tracy Mak, MD'3 , Justin Granstein, $\mathrm{MPH}^{\prime}$, and \\ Arnold Lefkowitz, PhD ${ }^{4}$
}

\begin{abstract}
This small-scale pilot study studied the potential benefit of taking a concentrated dose of anthocyanin $300 \mathrm{mg}$ once a day for 3 weeks (purple corn extract) on blood pressure among untreated Peruvian adults with mild-to-moderate hypertension. A double-blind placebo-controlled randomized crossover trial using intention-to-treat analysis was conducted on 30 healthy men and women who received care at the Dos de Mayo Hospital (Lima, Peru) between September 20 I0 and August 20I I. The 4-phase study compared blood pressure readings on placebo and on purple corn extract. Blood pressure readings decreased from baseline levels to end of study, especially after taking a purple corn extract capsule for 3 weeks, regardless of age, gender, body mass index level, or initial average blood pressure reading. Individuals who had higher blood pressure readings at baseline showed the most marked reduction in systolic and diastolic readings. Additional study of the possible mechanisms of action and the pharmacokinetics of purple corn extract warrants further investigation.
\end{abstract}

\section{Keywords}

anthocyanins, blood pressure, purple corn extract, hypertension, polyphenols

\section{Introduction}

Polyphenols, pigmented compounds found in many plants, and anthocyanins, a subcategory of polyphenols, have been the focus of numerous studies designed to assess their antioxidant, anticancer, and anti-inflammatory benefits. ${ }^{1}$ Anthocyanins, members of the flavonoid group of phytochemicals, are water-soluble vacuolar pigments that may appear red, purple, or blue depending on the $\mathrm{pH}$ value and the presence of copigments. The benefits of eating anthocyanin-rich foods have been linked to the prevention or delay of the onset of degenerative diseases of aging, ${ }^{2,3}$ cardiovascular disease, ${ }^{4,5}$ diabetes, ${ }^{6}$ cognitive dysfunction, ${ }^{7}$ cancer, ${ }^{8}$ act as an enhancement to the immune system, ${ }^{9}$ and as an antihypertensive agent. ${ }^{10}$ Although some of these health claims might be inflated or based on poorly designed studies, this apparently has not had an effect on the commercialization of anthocyanin-rich products; for example, marketing of acai as a health food.

The physiological and biochemical role of anthocyanins in the regulation of blood pressure, in particular, has been studied on animals. ${ }^{11,12}$ Findings from both studies showed that systolic and diastolic blood pressure among the rats in the purple corn extract experimental group was significantly lower than that for the control group, suggesting that anthocyanins had antihypertensive effects on hypertensive animals. Pertinent to any study that would include humans, the animal studies neither showed evidence of anthoycanin toxicity or its mutagenicity at any dose level nor was there evidence of adverse effects, even at the highest levels. ${ }^{13}$

Very few studies have been conducted on humans. One small-scale double-blind randomized placebo-controlled crossover study $(\mathrm{n}=31$ Norwegian males with screening blood pressure of greater than 140/90 mm Hg who were not on any antihypertensive or lipid-lowering medication) looked at whether high concentrations of anthocyanins (320 mg twice daily) would reduce blood pressure and alter cardiovascular and catecholamine reactivity to stress. The participants were followed for 4 weeks with a 4 -week washout. Findings showed no significant differences in blood pressure, no significant differences in stress reactivity, and no difference in semiautomatic oscillometric blood pressure measurements. ${ }^{14}$ Another study by the same researchers and with the same study sample found

\footnotetext{
'Weill Cornell Medical College, New York, NY, USA

${ }^{2}$ Universidad San Martin de Porres, AC PROESA, Lima, Peru

${ }^{3}$ Baylor Medical Center, Houston, TX, USA

${ }^{4}$ Armonk, NY, USA

Corresponding Author:

Madelon L. Finkel, PhD, Weill Cornell Medical College, 402 E 67 Street, New York, NY 10065, USA.

Email:maf20II@med.cornell.edu
} 
no beneficial effects in the short term on pathophysiological markers of cardiovascular disease. ${ }^{15}$

\section{Purpose of Study}

This small-scale pilot study sought to empirically study whether taking a concentrated dose of anthocyanin would have an effect on blood pressure. Of all purple-colored fruits and vegetables, Purple corn (Zea mays L.), cultivated in Peru and Bolivia and a staple in the Peruvian diet, contains the highest concentration of anthocyanins. ${ }^{16,17}$ We sought to test empirically the effect of purple corn extract on blood pressure among individuals with prehypertension (systolic 120-139 mm Hg; diastolic $80-89 \mathrm{mmHg}$ ) or stage 1 hypertension (systolic 140-159 mm Hg; diastolic 90-99 mmHg) who are not currently on antihypertensive medication. Estimates of the prevalence of hypertension in Peru range from $16 \%$ to $31 \%$ among men and from $15 \%$ to $23 \%$ among women. ${ }^{18,19}$ Many Peruvians with mild or elevated hypertension are not taking antihypertensive medication. It was hypothesized that taking a daily capsule of purple corn extract $(300 \mathrm{mg})$ would reduce both systolic and diastolic blood pressure among untreated individuals with prehypertension and stage 1 hypertension.

\section{Materials and Methods}

\section{Participants}

Healthy volunteers seeking care at the Cardiology Clinic of the Dos de Mayo Hospital in Lima, Peru were screened for inclusion in the study. Males and females between the ages of 21 and 70 years with prehypertension or stage 1 hypertension (based on an average of 3 blood pressure readings taken at the hospital outpatient clinic) were considered eligible to participate in the study. Excluded were individuals who were taking prescription antihypertension medication as well as those individuals who currently have diabetes, coronary heart disease, cancer, or uncontrolled or severe hypertension $(>160 />90 \mathrm{~mm} \mathrm{Hg})$. Women who were currently pregnant or intending to become pregnant within the year were excluded, and those who were deemed unable to understand the intent of the study and/or follow the research protocol, including making a commitment to come to the clinic to have their blood pressure taken at specified intervals, were excluded. A total of 30 individuals were included in the study.

The purpose of the study was orally explained, including a discussion of the potential benefits of taking purple corn extract capsules (ie, reduction in blood pressure) and possible risks (ie, inability to lower blood pressure to normal levels). A written document delineating the purpose, potential benefits, and possible risks of the study was handed to the individual. Taxi fare was provided to each participant for the multiple trips to and from the hospital. The Dos de Mayo Hospital Institutional Review Board approval was obtained. A small pilot study to assess feasibility of conducting this project was conducted by a medical student (TM).

\section{Purple Corn Extract Capsule}

Fitofarma, located in Lima, Peru, is a major global manufacturer of liquid and powered extracts from Peruvian herbs, fruits, and vegetables, including purple corn. Fitofarma produces capsules of purple corn extract in a soft gelatin casing; a typical number " 0 " size capsule holds $300 \mathrm{mg}$ of purple corn extract in a maltodextrin carrier, which is $15 \%$ by weight. There is less than $1 \%$ silica and magnesium stearate, which is used to facilitate the flow of the powder. The final product contains $6 \%$ of total anthocyanin $(6 \mathrm{mg}$ anthocyanin per $100 \mathrm{mg}$ of purple corn extract) and $15 \%$ phenolic compounds. ${ }^{20}$ In 2003, Fitofarma had its purple corn extract product undergo chemical analysis and evidence of safety. Findings showed that the main ingredient in the purple corn extract was carbohydrates $(76.6 \%)$; there was a low (8\%) moisture content, and negligible amounts of fat $(0.13 \%)$ and protein $(0.59 \%)$. The sodium ion level was $2.58 \%$ and the magnesium ion level was $<2 \%$. Findings showed no measurable amounts of any metals or pesticides. Arsenic, cadmium, lead, and mercury were not found in detectable amounts in purple corn extract. ${ }^{21}$

In 2005, as part of a New Dietary Ingredient application to the US Food and Drug Administration, a toxicity screen was performed. Findings showed no evidence of human or animal toxicity, mutagenicity, or cytotoxicity to healthy cells. As part of the analysis, in 2005 a search for evidence of toxicity from "purple corn" and "purple corn extract" was made in PubMed, TOXNET, EMBASE, and BIOSIS. No evidence of human or animal toxicity, mutagenicity, or cytotoxicity to healthy cells was found. ${ }^{22}$ Based on results from the literature search, animal studies and the toxicity studies, the evidence convincingly show no toxic effects of purple corn extract or anthocyanin even at very high levels. Fitofarma prepared the capsules containing purple corn extract and identical placebo capsules in size and color but contained no anthocyanin or phenolic compounds.

\section{Study Design}

A double-blinded placebo-controlled randomized crossover trial using intention-to-treat analysis was conducted. Using a block randomization scheme, patients were randomized to 1 of 2 groups: taking the placebo capsule before taking the purple corn extract capsule or to taking the purple corn extract capsule first and then the placebo. The study was administered by nurse project assistant based at the Dos de Mayo Hospital who attended a training session organized by the Research Coordinator and Peruvian Principal Investigator (SS) to ensure standardized administration of the study protocol. Participants were enrolled between September 2010 and August 2011.

After eligibility was determined and consent obtained, a baseline survey designed to obtain demographic and medical history was administered by a nurse project assistant who recorded answers from each participant on to a standardized coding form. All participants were seen during the morning between $9 \mathrm{AM}$ and $11 \mathrm{AM}$. Weight, height, and blood pressure (taken 3 times in both sitting and supine positions within a few minutes to obtain an average baseline reading) were measured according to a standard protocol. Blood pressure was taken by the same individual in a standardized fashion using a sphygmomanometer. Body mass index was calculated as weight in kilograms divided by the square of height in meters. Demographics, reproductive history, family history of high blood pressure, smoking, and alcohol consumption were recorded.

There were 4 phases to this study (see Table 1 for time frame and randomization scheme). During phase 1 (3 weeks in length), every participant was given a 2-week supply of the placebo capsule and told to take one each day in the morning (at breakfast time). At the end of 2 weeks, the participants were asked to return to the clinic to have their blood pressure taken as described above. A short questionnaire elicited information about how they have been feeling and if they experienced any adverse health effects during the past 2 weeks. The 
Table I. Randomization Scheme and Timeline.

\begin{tabular}{|c|c|c|}
\hline & $\begin{array}{l}\text { Group I } \\
(\mathrm{n}=18)\end{array}$ & $\begin{array}{l}\text { Group } 2 \\
(\mathrm{n}=12)\end{array}$ \\
\hline $\begin{array}{l}\text { Phase I: Baseline ( } 3 \text { weeks) } \\
\text { Groups randomized to purple } \\
\text { corn extract or placebo }\end{array}$ & Placebo & Placebo \\
\hline Phase 2 ( 3 weeks) & $\begin{array}{l}\text { Purple corn } \\
\text { extract capsule }\end{array}$ & Placebo \\
\hline $\begin{array}{l}\text { I Week washout period-no } \\
\text { capsules consumed }\end{array}$ & & \\
\hline Phase 3 ( 3 weeks) & Placebo & $\begin{array}{l}\text { Purple corn } \\
\text { extract capsule }\end{array}$ \\
\hline Phase 4 (2 weeks) & $\begin{array}{l}\text { No capsules } \\
\text { consumed }\end{array}$ & $\begin{array}{l}\text { No capsules } \\
\text { consumed }\end{array}$ \\
\hline
\end{tabular}

participants were then given a 7-day supply of the placebo capsules and asked to return in one week where the same process as just described was repeated. If an individual's blood pressure increased to a level that warranted medical intervention at any time during the study, the individual would be censored (excluded from the study) and referred to a physician for treatment.

At the end of the 3-week period of phase 1, based on the randomization scheme, participants either received another round of the placebo capsule or the purple corn extract capsule. During this phase 2 period, participants returned to the clinic after 2 weeks at which time blood pressure was taken in the manner described above and the short questionnaire orally administered to elicit any adverse health effects experienced over the past 2 weeks. Participants were then given a 7-day supply of the capsules and asked to return in a week at which time the same process as described above was repeated. Compliance was assessed by pill count.

A wash out period of 1 week where no capsules were consumed followed phase 2. Individuals returned to the clinic the following week.

At phase 3, which also lasted 3 weeks, those who had been taking the purple corn extract capsule were given the placebo and those who had been taking the placebo were given the purple corn extract capsule. The same process described for phase 2 was repeated in phase 3 . In phase 4, which lasted 2 weeks, no capsules were consumed. Blood pressure was measured and a short questionnaire was administered orally in the manner described above.

\section{Statistical Considerations}

We hypothesized that the purple corn extract supplement would be more effective than placebo in reducing blood pressure. Comparisons of average blood pressure readings were made for each participant at the end of each phase. Baseline characteristics were summarized for the total sample population and by group. A paired 2-tailed $t$ test statistic was used to test for statistical significance in blood pressure readings. A $P$ value of less than .05 was considered statistically significant. This was a pilot study of 30 individuals and power calculations were not made as we could not assume what would be an appropriate reduction in blood pressure since there have not been any studies done on humans.

\section{Results}

Table 2 presents the demographic and selected health characteristics of the sample. Two thirds of the sample (63.3\%) was
Table 2. Demographic and Selected Health Characteristics of Sample.

\begin{tabular}{|c|c|c|}
\hline Variable & $\mathrm{n}$ & Percentage \\
\hline \multicolumn{3}{|l|}{ Gender } \\
\hline Male & $\mathrm{II}$ & 36.7 \\
\hline Female & 19 & 63.3 \\
\hline \multicolumn{3}{|l|}{ Age (years) } \\
\hline$<35$ & 2 & 6.7 \\
\hline $35-44$ & 4 & 13.3 \\
\hline $45-54$ & 10 & 33.3 \\
\hline $55-64$ & II & 36.7 \\
\hline$\geq 65$ & 3 & 10.0 \\
\hline \multicolumn{3}{|l|}{ Level of education } \\
\hline Primary school & 6 & 20.0 \\
\hline Secondary school & 6 & 20.0 \\
\hline Technical school & 16 & 53.3 \\
\hline University & 2 & 6.7 \\
\hline \multicolumn{3}{|c|}{ Body mass index $\left(\mathrm{kg} / \mathrm{m}^{2}\right)$} \\
\hline$<25$ & 3 & 10.0 \\
\hline $25-29$ & 20 & 66.7 \\
\hline $30-34$ & 6 & 20.0 \\
\hline$\geq 35$ & I & 3.3 \\
\hline \multicolumn{3}{|c|}{ Ever told have hypertension } \\
\hline Yes & 20 & 66.7 \\
\hline No & 10 & 33.3 \\
\hline \multicolumn{3}{|l|}{ Smoking status } \\
\hline Never & 20 & 66.7 \\
\hline Former & 6 & 20.0 \\
\hline Current & 4 & 13.3 \\
\hline \multicolumn{3}{|l|}{ Drink Chicha Morada } \\
\hline Never & 3 & 10.0 \\
\hline Yes, in past & 15 & 50.0 \\
\hline Yes, currently & 12 & 40.0 \\
\hline
\end{tabular}

women and the majority of participants $(70 \%)$ were middleaged (45-64 years). Sixty percent were graduates of a technical school or university whereas $20 \%$ had a primary school education or secondary school education. Two thirds $(66.7 \%)$ had a body mass index between 25 and $29 \mathrm{~kg} / \mathrm{m}^{2}$ and $20 \%$ had a body mass index between 30 and $35 \mathrm{~kg} / \mathrm{m}^{2}$. Three individuals had a body mass index less than $25 \mathrm{~kg} / \mathrm{m}^{2}$ and one person had a body mass index greater than $35 \mathrm{~kg} / \mathrm{m}^{2}$. There were no statistically significant differences between those who were randomized to the experimental group and those to the placebo group. Each individual was serving as his or her own control.

The majority $(66.7 \%)$ reported that they never smoked tobacco; only 4 individuals currently smoked cigarettes. Almost half (46.7\%) reported that they never drank alcohol and none reported using illicit drugs. Three quarters $(76.7 \%)$ of the sample reported that they were not taking prescription medications. Of the 7 individuals who were on medication, none were taking pills for hypertension. However, two thirds (66.7\%) reported that in the past they had been told that they had high blood pressure (55\% between the age of 50 and 60 years, and $25 \%$ between the ages of 45 and 49 years).

Regarding family history of hypertension, $60 \%$ said that their mother had hypertension, $16.7 \%$ said that their father had high blood pressure, and $36.7 \%$ said that they had siblings 
Table 3. Blood Pressure at Baseline, After Taking Purple Corn Extract Capsule, and at End of Study, by Group. ${ }^{a}$

\begin{tabular}{|c|c|c|c|c|c|c|}
\hline & \multicolumn{2}{|c|}{$\begin{array}{l}\text { Group I } \\
(n=18)\end{array}$} & \multicolumn{2}{|c|}{$\begin{array}{l}\text { Group } 2 \\
(n=12)\end{array}$} & \multicolumn{2}{|c|}{$\begin{array}{c}\text { Total } \\
(\mathrm{n}=30)\end{array}$} \\
\hline & $\bar{x}$ & SD & $\bar{x}$ & SD & $\bar{x}$ & SD \\
\hline \multicolumn{7}{|c|}{ Baseline (all on placebo) } \\
\hline Systolic & 137.2 & 7.2 & $14 \mid .7$ & 7.0 & 139.0 & 7.4 \\
\hline Diastolic & 87.2 & 5.1 & 88.5 & 8.6 & 87.7 & 6.6 \\
\hline \multicolumn{7}{|c|}{ After taking purple corn extract } \\
\hline Systolic & 131.6 & 5.8 & 129.3 & 7.7 & 130.7 & 6.6 \\
\hline Diastolic & 81.7 & 4.9 & 77.9 & 7.3 & 80.2 & 6.1 \\
\hline \multicolumn{7}{|c|}{ End of phase 4 (all on placebo) } \\
\hline Systolic & $13 \mid .5$ & 6.9 & 133.8 & 5.6 & 132.4 & 6.4 \\
\hline Diastolic & 81.7 & 6.1 & 80.2 & 7.9 & 81.1 & 6.8 \\
\hline
\end{tabular}

${ }^{a}$ One individual dropped out after taking the purple corn extract capsule but did not complete the last placebo phase.

who were diagnosed with high blood pressure. A very small minority reported that an immediate family member had had a stroke or heart attack. Diabetes appeared not to be a medical problem among the respondents or among their immediate family.

\section{Effect of Purple Corn Extract on Blood Pressure}

Mean systolic and diastolic blood pressure at baseline for the entire sample was 139 and $87.7 \mathrm{~mm} \mathrm{Hg}$, respectively, with a systolic range of 122-155 $\mathrm{mm} \mathrm{Hg}$ and a diastolic range of 65-97 mm Hg. Median baseline systolic pressure was 139.5 $\mathrm{mm} \mathrm{Hg}$ and median baseline diastolic pressure was $90 \mathrm{~mm}$ Hg. Mean systolic and diastolic pressure after taking the purple corn extract capsule was 130.7 and $80.2 \mathrm{~mm} \mathrm{Hg}$, respectively. At the end of the study, the systolic and diastolic pressure readings were lower than at baseline $(139 \mathrm{~mm} \mathrm{Hg}$ at baseline compared with $132.4 \mathrm{~mm} \mathrm{Hg}$ at the end of study and $87.7 \mathrm{~mm} \mathrm{Hg}$ at baseline compared with $81.1 \mathrm{~mm} \mathrm{Hg}$ at the end of the study). Individually, the biggest drop in systolic pressure from baseline to end of study was from 154 to $132 \mathrm{~mm} \mathrm{Hg}$, a 22-point drop; the biggest drop in diastolic pressure was from 95 to $73 \mathrm{~mm} \mathrm{Hg}$, a 22 point drop. No individual reported any adverse effects from taking the purple corn extract capsule.

The subjects were not randomized by blood pressure reading. Group 1 was randomized to placebo, capsule, placebo, and group 2 to placebo, placebo, capsule. All participants consumed no capsules thereafter for 2 weeks. Table 3 shows blood pressure readings at baseline, after taking purple corn extract capsules, and at the end of study. Baseline mean blood pressure reading for group 1 was 137.2/87.2 $\mathrm{mm} \mathrm{Hg}$ (baseline median blood pressure readings was $135 / 85.5 \mathrm{~mm} \mathrm{Hg}$ ) compared with group 2's mean readings of 141.7/88.5 mm Hg (median readings of 142/93 mm Hg). However, every individual, regardless of which group he or she was randomized to, showed a decrease in blood pressure reading from baseline to end of taking the purple corn extract capsule.
For those in group 1, a paired $t$ test showed statistically significant differences in systolic and in diastolic readings from baseline to end of study $(P<.001)$. Blood pressure dropped while on the purple corn extract capsule and at the end of the study, the blood pressure readings remained lower than at the beginning of the study $(P<.001)$. Specifically, mean baseline systolic pressure decreased from 137.2 to $131.6 \mathrm{~mm} \mathrm{Hg}$ after taking the purple corn extract capsule. At end of study, the mean systolic pressure was $131.5 \mathrm{~mm}$ $\mathrm{Hg}$. Mean baseline diastolic pressures were 87.2 and 81.7 $\mathrm{mm} \mathrm{Hg}$ after taking the purple corn extract capsule. At end of the study, mean diastolic pressure was $81.7 \mathrm{~mm} \mathrm{Hg}$. Among this group, there was a 5.6-point drop in systolic blood pressure from baseline compared with that taken at end of the 3 -week period in which the purple corn extract capsule was taken. There was a 5.5 point drop in diastolic blood pressure. Blood pressure remained significantly lower at the end of the study.

Individuals in group 2 took the purple corn extract capsule after 6 weeks on placebo whereas group 1 took the capsule between weeks taking the placebo. For those in group 2, a paired $t$ test showed statistically significant differences in systolic and in diastolic readings from baseline to end of study $(P<.001)$. Blood pressure dropped while on the purple corn extract capsule and at the end of the study the blood pressure readings remained lower than at the beginning of the study $(P<.001)$. Specifically, mean baseline systolic pressure decreased from $141.7 \mathrm{~mm} \mathrm{Hg}$ to an impressive $129.3 \mathrm{~mm} \mathrm{Hg}$ after taking the purple corn extract capsule, a drop of 12.4 points. At end of the study, mean systolic pressure was 133.8 $\mathrm{mm} \mathrm{Hg}$. Mean baseline diastolic pressure was $88.5 \mathrm{~mm} \mathrm{Hg}$ and after taking the purple corn extract capsule the mean was 77.9 $\mathrm{mm} \mathrm{Hg}$, a drop of 10.6 points. At end of the study, mean diastolic pressure was $80.2 \mathrm{~mm} \mathrm{Hg}$. One individual dropped out after taking the purple corn extract capsule and did not complete the last placebo phase.

\section{Discussion}

This small pilot study showed that among participants with prehypertension or stage 1 hypertension, taking a purple corn extract capsule for a short period of time (3 weeks) apparently had a beneficial effect of reducing systolic and diastolic blood pressure. None of the individuals had been taking antihypertension medication for their blood pressure. Study findings showed that blood pressure readings for every individual, regardless of age, gender, body mass index level, or initial average blood pressure reading, decreased from baseline levels to end of study, especially after taking a purple corn extract capsule for 3 weeks. In this study, those individuals who had higher blood pressure readings at baseline showed the most marked reduction in systolic and diastolic readings during which the purple corn extract capsule was taken as well as at the end of the study; however, end-of-study blood pressure readings were starting to creep up among those in group 2. That is, lowest blood pressure readings were at the end of taking the 
purple corn extract capsule but apparently were increasing (not statistically or clinically) by the end of the study. Given that many Peruvians with mildly elevated blood pressure do not take medication, having these individuals take a daily capsule of purple corn extract might help keep their blood pressure in check over time.

This pilot study had several limitations, including the small sample size and the fact that the individuals took the purple corn extract capsule for a very short period of time ( 3 weeks). It would have been interesting to have followed the participations for a longer period of time to determine if, after taking the purple corn extract capsule, their blood pressure would have increased. Given the findings from this small study, it might be instructive to follow a larger group of individuals for a longer period of time to assess the effectiveness of purple corn extract on blood pressure. Furthermore, additional study of the possible mechanisms of action and the pharmacokinetics of purple corn extract and/or anthocyanins in general warrant investigation. The $300 \mathrm{mg}$ capsule contained a very small amount of anthocyanins (6\%). It would be of interest to see what effect a larger dose of anthocyanins would have on blood pressure over time.

\section{Conclusion}

The findings of this small pilot study are intriguing. Given the small sample and short period during which the participants were taking the purple corn extract capsule, it is unclear what the true independent treatment effect was. The study findings imply that a larger study would be warranted to help better understand the longer term effect of taking purple corn extract over time among individuals with prehypertension who are not on medication.

\section{Acknowledgments}

We gratefully thank Dr Carlos Pino, Chief Department of Cardiology of the Dos de Mayo Hospital for allowing the study to be conducted at his clinic, and Roberto Culloti of FitoFarma for providing the purple corn extract and placebo capsules for this study. Many thanks to Elena Sanchez for coordinating the study and Rebecca Vergara for administering the survey questionnaires.

\section{Author Contributions}

Each author contributed equally to the design of the study and the preparation of the manuscript. Dr Finkel wrote the first draft of the article, and Dr Finkel, Dr Mak, and Mr Granstein analyzed the data.

\section{Declaration of Conflicting Interests}

The authors declared no potential conflicts of interest with respect to the research, authorship, and/or publication of this article.

\section{Funding}

The authors received no financial support for the research, authorship, and/or publication of this article.

\section{Ethical Approval}

The Dos de Mayo Hospital Institutional Review Board approved this study.

\section{References}

1. Wang H, Cao G, Prior RL. Oxygen radical absorbing capacity of anthocyanins. J Agric Food Chem. 1997;45:304-309.

2. Casto BC, Kresty LA, Kraly CL, et al. Chemoprevention of oral cancer by black raspberries. Anticancer Res. 2002;22:4005-4015.

3. Zhao C, Giusti MM, Malik M, Moyer MP, Magnuson BA. Effects of commercial anthocyanin-rich extracts on colonic cancer and nontumorigenic colonic cell growth. J Agric Food Chem. 2004; 52:6122-6128.

4. Heinonen IM, Meyer AS, Frankel EN. Antioxidant activity of berry phenolics on human low-density lipoprotein and liposome oxidation. J Agric Food Chem. 1998;46:4107-4112.

5. Pawlowicz P, Wilczynski J, Stachowiak G, Hincz P. Administration of natural anthocyanins derived from chokeberry retardation of idiopathic and preeclamptic origin. Influence on metabolism of plasma oxidized lipoproteins: the role of autoantibodies to oxidized low density lipoproteins. Ginekol Pol. 2000;71:848-853.

6. McDougall GJ, Stewart D. The inhibitory effects of berry polyphenols on digestive enzymes. Biofactors. 2005;23:189-195.

7. Andres-Lacueva C, Shukitt-Hale B, Galli R, Jauregui O, Lamuela-Raventos R, Joseph JA. Anthocyanins in aged blueberry-fed rats are found centrally and may enhance memory. Nutr Neurosci. 2005;8:111-120.

8. Wang SL, Stoner GD. Anthocyanins and their role in cancer prevention. Cancer Lett. 2001;171:17-25.

9. Bub A, Watzl B, Blockhaus M, et al. Fruit juice consumption modulates antioxidative status, immune status and DNA damage. J Nutr Biochem. 2003;14:90-98.

10. Carr A, Frei B. The role of natural antioxidants in preserving the biological activity of endothelium-derived nitric oxide. Free Radic Biol Med. 2000;28:1806-1814.

11. Toyoshi T, Kohda T. Antihypertensive activity of purple corn color in spontaneously hypertensive rats. Foods Food Ingredients J Japan. 2004;209:676-679.

12. Shindo M, Kasai T, Abe A, Konido Y. Effects of dietary administration of plant-derived anthocyanin-rich colors to spontaneously hypertensive rats. J Nutr Sci Vitaminol (Tokyo). 2007; 53:90-93.

13. Biological Test-Acute Toxicity. Universidad Peruana Cayetano Heredia. Quality Control Services. Assay Report \#P0015799/02. October 18, 2002.

14. Hassellund SS, Flaa A, Sandvik L, Kjeldsen SE, Rostrup M. Effects of anthocyanins on blood pressure and stress reactivity: a double-blind randomized placebo controlled crossover study. J Hum Hypertens. 2012;26:396-404.

15. Hassellund SS, Flaa A, Kjeldsen SE, et al. Effects of anthocyanins on cardiovascular risk factors and inflammation in prehypertensive men: a double-blind randomized placebocontrolled crossover study. J Hum Hypertens. 2012;26:1-7.

16. Aoki H, Kuze N, Kato Y. Anthocyanins isolated from purple corn (Zea mays L.). Foods Food Ingredients J Japan. 2002;199:41-45. 
17. Jing P. Purple Corn Anthocyanins: Chemical Structure, Chemoprotective Activity and Structure/Function Relationships [dissertation]. Columbus, OH: The Ohio State University; 2006.

18. Davies AR, Miranda JJ, Gilman RH, Smeeth L. Hypertension among adults in a deprived urban area of Peru-undiagnosed and uncontrolled? BMC Res Notes. 2008;26:1-2.

19. Goldstein J, Jacoby E, del Aguila R, Lopez A. Poverty is a predictor of non-communicable disease among adults in Peruvian cities. Prev Med. 2005;41:800-806.
20. Chemical analysis of purple corn extract for Laboratorios Fitofarma. International Analytical Services. SAC. Lima, Peru. November 17, 2003.

21. Heavy metals and pesticides assay of purple corn extract for Laboratorios Fitofarma. International Analytical Services. SAC. Lima, Peru. September 8, 2003.

22. Purple corn extract. NDI Application submitted by Rainforest Botanicals LCC to the Office of Nutritional Products, Labeling, and Dietary Supplements. Center for Food Safety and Applied Nutrition. Food and Drug Administration. February 28, 2005. 